Research Article

\title{
Dentogingival Smile Analysis of Young Adults of Al Qassim Province, Saudi Arabia: A Cross-Sectional Study
}

\author{
Minu P. Mohan $\left(\mathbb{D},{ }^{1}\right.$ Rahaf A. Alolayan ${ }^{(D},{ }^{2}$ and Mohammad A. AlSweed $\mathbb{D}^{3}$ \\ ${ }^{1}$ Assistant Professor, Department of Prosthetic Dental Sciences, College of Dentistry, Al Qassim University, \\ Buraydah 51451, Saudi Arabia \\ ${ }^{2}$ Dental Intern, College of Dentistry, Al Qassim University, Buraydah 56219, Saudi Arabia \\ ${ }^{3}$ General Practitioner, Private Practice, Buraydah 52353, Saudi Arabia \\ Correspondence should be addressed to Minu P. Mohan; dr.minu.mohan@qudent.org
}

Received 21 August 2020; Revised 21 October 2020; Accepted 24 October 2020; Published 11 November 2020

Academic Editor: Andrea Scribante

Copyright (c) 2020 Minu P. Mohan et al. This is an open access article distributed under the Creative Commons Attribution License, which permits unrestricted use, distribution, and reproduction in any medium, provided the original work is properly cited.

\begin{abstract}
Objectives. The present study aims to analyse the smiling components among young adults within $\mathrm{Al}$ Qassim region by evaluating typical smile parameters. Methodology. A cross-sectional study was conducted among 324 female and male participants between 18 and 35 years, within Al Qassim Province of Saudi Arabia. The dentogingival macro-aesthetic elements of the smile, the parallelism between the incisal curve and lower lip line, midline, and buccal corridors were determined by using Canon Rebel t7i (Canon, Melville, NY, USA) and evaluated by photo editing software Adobe Photoshop CC2019. The data were analysed using a chi-square test and Spearman's correlation test for nonparametric data. Results. $62.5 \%$ of young adults had no buccal corridors. The nonparallel smile was found in $53.2 \%$ of young adults. A high smile line was observed in $33 \%$ of participants. 59.3\% of young adults lacked a coinciding dental and facial midline. There was a statistically significant difference in the parallelism of the incisal curve and lower lip line as well as in the position of the upper lip line across genders $(P<0.05)$. Conclusion. Understanding macroaesthetic elements of teeth and their interrelation with the surrounding oral structures can be a guide in creating natural and aesthetically pleasing restorative treatment.
\end{abstract}

\section{Introduction}

Smiling is one of the most critical facial expressions and is known as a nonverbal parameter of correspondence [1]. Cosmetic dentistry plays a significant role in improving the smile of a patient. A systematic and comprehensive dentofacial analysis must be performed before commencing aesthetic treatment [2]. Creating an aesthetically pleasing smile requires the integration of facial and dental parameters. Dental and facial aesthetics can be defined in terms of macro- and microelements [3]. Macro-aesthetic components include interrelationships between the face, lips, gingiva, and teeth. The aesthetics of an individual tooth and its color and form comprise micro-aesthetics [3]. Analysis of smile integrates facial, dentofacial, and dental aesthetics, encompassing the macro- and microelements [3].
Several studies involving different populations have provided evidence that aesthetic components for different sexes, races, and ages are not entirely the same [4-6]. However, the number of studies conducted to assess smile parameters within the Saudi population is minimal $[6,7]$. Additionally, results of these studies were variable and inconclusive. Furthermore, none of these studies were conducted within Al Qassim region to assess and analyse different smile parameters $[6,7]$.

Appreciation of what society considers acceptable and aesthetically pleasing is crucial for a successful outcome of prosthetic and restorative treatment $[8,9]$. The characteristic feature of a smile, described by several researchers, has served as a guideline for restoration enhancement for the anterior component of the dentition [8, 10-12]. Smile analysis-obtaining averages of the smile's characteristic 
features among various populations-provides an understanding of these features' general pattern of occurrence [12].

Tjan et al. formulated a standard of normalcy in an aesthetic smile relative to the smiling type: high, average, and low; parallelism of the maxillary incisal curve with the lower lip; and the number of teeth seen when smiling [1].

The buccal corridor is a significant aesthetic parameter [13-16]. It is defined as the negative spaces between the facial surfaces of the posterior teeth and the commissure of the mouth when the patient smiles. The buccal corridor space's width influences smile attractiveness in different facial types [15].

The coincidence of the dental midline and the facial midline is a controversial subject in aesthetics, and many researchers suggest that it is more important for the facial midline and the maxillary dental midline to coincide rather than the facial and mandibular midlines. The dominant visibility of the maxillary teeth when smiling is a primary reason behind this argument [17-19]. The coincidence of facial, maxillary, and mandibular midlines is favourable but not mandatory [17]. In recent years, attempts to include smile analysis and smile aspects into treatment planning have become the key to prosthodontic treatment success $[12,20,21]$.

The null hypothesis of this study is that there would be no difference in the distribution and analysis of smile parameters among young Saudis within Al Qassim Province. A cross-sectional study was conducted to assess the dentalgingival macro-aesthetic elements of a smile, the position of the upper lip line, the parallelism of the incisal line and lower lip line, location of the arch midline, and presence or absence of the buccal corridor among participants. Findings may be used as a guide for advanced smile designing and restorative procedures for this population.

\section{Materials and Methods}

The study was approved by the ethical committee at $\mathrm{Al}$ Qassim University, KSA (SRPSSC), approval number: F-2018-3018, registered on 29 September 2018 following the ethical principles of research involving human subjects.

The cross-sectional study was conducted within Buraydah, Unizah, and Arras regions of Al Qassim Province. A pilot study was conducted previously to calculate the sample size. The total sample size contained 324 participants, comprising 162 males and 162 females, who reported to 10 dental clinics across Al Qassim Province. Informed consent was obtained from all 324 participants. The target study population included Saudi young adults between 18 and 35 years. Non-Saudi participants were excluded from the study. The present study also excluded participants who had prosthodontic crowns or orthodontically modified dentition. In fact, both prosthodontic frameworks and orthodontic appliances can alter aesthetic appearances [22, 23]. Additionally, participants who had bleached dentition, dental fluorosis, and hypoplasia were excluded from the study.
2.1. Photography. A standardized photograph (frontal view) was taken of each participant's full social smile (portrait) by a trained photographer, using Canon Rebel t7i (Canon, Melville, NY, USA). The settings were standardized to $1 / 60$ second shutter speed, F5.6, and ISO 400. A macroring flash (Yongnuo YN-14EX) was attached to the lens for standardized lighting. Canon tripod was adjusted individually according to the participant's height. The participant was seated at a $90^{\circ}$ head position on the dental chair. The photographs were captured $25 \mathrm{~cm}$ away from the participant's nose [24].

2.2. Smile Analysis. To assess the dentogingival components of the smile, Adobe Photoshop CC2019 (Adobe Inc, CA, USA) was used. The position of the upper lip line was located by drawing a line following the upper lip contour and gingival zenith of the upper anterior teeth (teeth included from \#13 to \#23). The upper lip line was considered high when the observed distance between the gingival zenith and upper lip line was $2-3 \mathrm{~mm}$, medium when the display of 75-100\% clinical crown height was seen, and low when the upper lip covered $25 \%$ or more of the labial surface of the upper anterior teeth [7].

The parallelism between the incisal curve and the lower lip line was determined by drawing two curves. The facial midline was located by drawing a line through the base of the philtrum. The dental midline was located using a line drawn through the central incisors. The deviation of the dental midline from the facial midline was recorded independently in pixels using a ruler tool (Adobe Photoshop CC2019, Adobe Inc., CA, USA) and was then converted into millimeters. The presence or absence of the buccal corridor between the posterior teeth and the commissures of the mouth when smiling was evaluated using a magnetic lasso tool (Adobe Photoshop CC2019, Adobe Inc., CA, USA), following the buccal corridor area (Figures 1 and 2).

2.3. Statistics. SPSS (IBM SPSS V25, IBM Corporation, Chicago, IL, USA) was used for data analysis. A descriptive analysis was done for all aforementioned variables: the position of the upper lip, incisal line or parallelism, position of the midline, amount of shift, and presence or absence of the buccal corridor.

Chi-square, Mann-Whitney $U$, and independent $t$-tests were used to investigate if there was a significant difference between male and female smiles. For all statistical testing, $P=0.05$.

\section{Results}

The obtained data were arranged systematically, using SPSS V25. The collected information was transferred from a table, created with Excel 2016. Data from all 324 participants (162 females and 162 males) were analysed. The distribution of smile parameters among the participants is explained in Table 1. The plot of the mean score for each combination of groups of "gender" and "smile parameters" is presented using a line graph (Figure 3). 


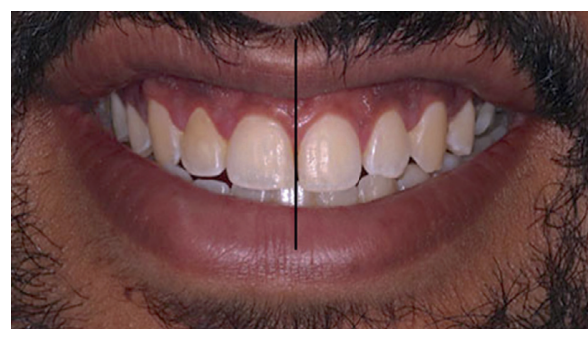

(a)

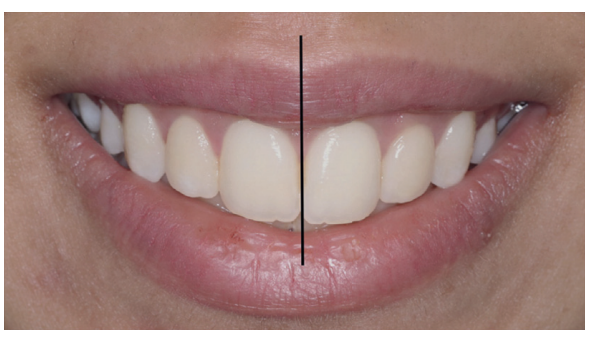

(b)

Figure 1: (a) Male subject showing no buccal corridor and no midline shift. (b) Female subject showing no buccal corridor and no midline shift.

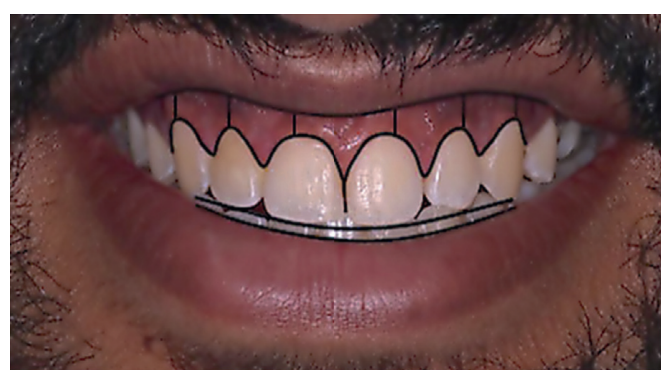

(a)

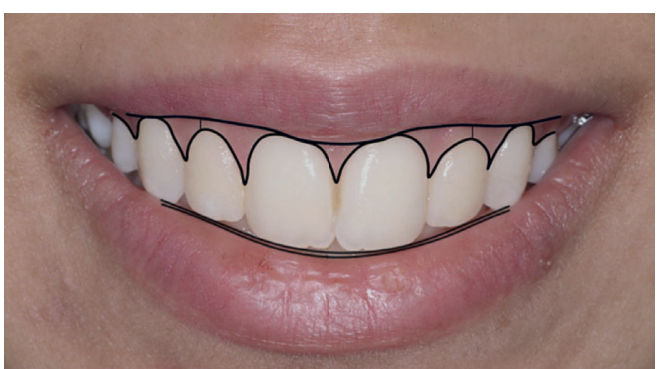

(b)

Figure 2: (a) Male subject showing parallel smile and high lip line. (b) Female subject showing parallel smile and medium lip line.

TABLE 1: Frequency distribution table of different smile parameters.

\begin{tabular}{lccc}
\hline & & Frequency & $\%$ \\
\hline \multirow{2}{*}{ Gender } & Female & 162 & 50.0 \\
& Male & 162 & 50.0 \\
\hline \multirow{2}{*}{ Midline shift } & $0-1 \mathrm{~mm}$ & 132 & 40.7 \\
& $\geq 2 \mathrm{~mm}$ & 192 & 59.3 \\
\multirow{2}{*}{ Lip line } & Low & 86 & 26.5 \\
& High & 107 & 33.0 \\
\multirow{2}{*}{ Parallelism between } & Medium & 131 & 40.4 \\
lower lip and smile line & No & 173 & 53.4 \\
\multirow{2}{*}{ Buccal corridor } & Yes & 151 & 46.6 \\
\hline
\end{tabular}

3.1. Smile Line. The distribution of female participants' smile line parameters was as follows: $51.2 \%$ with a medium smile line, $24.7 \%$ with a high smile line, and $24.1 \%$ with a low smile line. For male participants, $41.4 \%$ had a high smile line, $29.6 \%$ had a medium smile line, and $29 \%$ had a low smile line. There was a statistical difference between females and males in smile line distribution when using the Mann-Whitney $U$ test for nonparametric data (Table 2).

3.2. Buccal Corridor. Buccal corridors were absent in $65.4 \%$ of females and $59.9 \%$ of males. A chi-square test was applied to test the difference between males and females in the

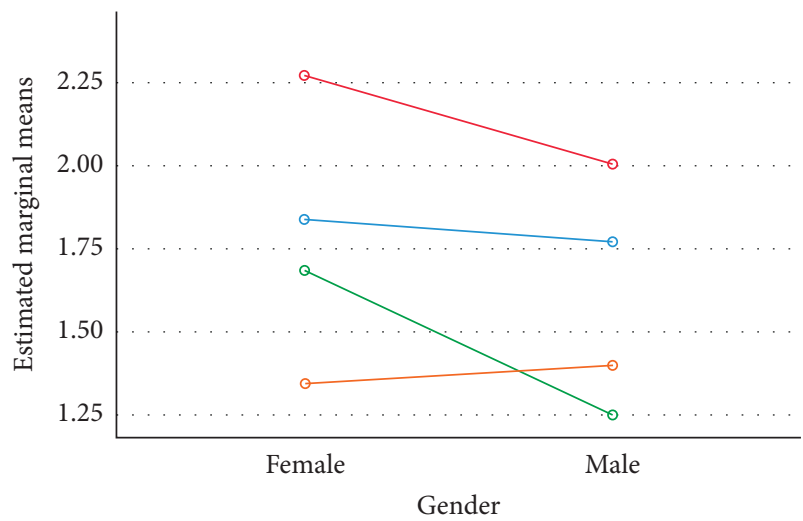

Smile parameters

- Midline shift _ Paralellism

— Lipline _ Buccal corridor

FIgURE 3: Plot of the mean score for smile parameters and gender.

presence or absence of a buccal corridor, and no statistical difference was found (Table 2).

3.3. Parallelism. The parallelism between the incisal curve and the lower lip line was classified as parallel and nonparallel. Parallelism was found in $68.5 \%$ of females and $24.7 \%$ of males, while $31.5 \%$ of females and $75.3 \%$ of males had nonparallel smiles. The chi-square test showed that the 
TABLE 2: Chi-square test for the association between gender and different smile parameters.

\begin{tabular}{|c|c|c|c|c|c|c|}
\hline & & & Female & Male & Pearson chi-square & $P$ value* \\
\hline \multirow{10}{*}{ Midline shift } & \multirow{2}{*}{0} & Count & 18 & 20 & \multirow{10}{*}{1.892} & \multirow{10}{*}{0.756} \\
\hline & & $\%$ within gender & 11.1 & 12.3 & & \\
\hline & \multirow{2}{*}{1} & Count & 43 & 51 & & \\
\hline & & $\%$ within gender & 26.5 & 31.5 & & \\
\hline & \multirow[b]{2}{*}{2} & Count & 57 & 48 & & \\
\hline & & $\%$ within gender & 35.2 & 29.6 & & \\
\hline & \multirow{2}{*}{3} & Count & 35 & 32 & & \\
\hline & & $\%$ within gender & 21.6 & 19.8 & & \\
\hline & \multirow{2}{*}{4} & Count & 9 & 11 & & \\
\hline & & $\%$ within gender & 5.6 & 6.8 & & \\
\hline \multirow{6}{*}{ Smile line } & \multirow{2}{*}{ Low } & Count & 39 & 47 & \multirow{6}{*}{16.908} & \multirow{6}{*}{$0.000^{* *}$} \\
\hline & & $\%$ within gender & 24.1 & 29.0 & & \\
\hline & & Count & 83 & 48 & & \\
\hline & Medium & $\%$ within gender & 51.2 & 29.6 & & \\
\hline & \multirow{2}{*}{ High } & Count & 40 & 67 & & \\
\hline & & $\%$ within gender & 24.7 & 41.4 & & \\
\hline \multirow{4}{*}{ Parallelism } & \multirow{2}{*}{ No } & Count & 51 & 122 & \multirow{4}{*}{62.523} & \multirow{4}{*}{$0.000^{* *}$} \\
\hline & & $\%$ within gender & 31.5 & 75.3 & & \\
\hline & \multirow{2}{*}{ Yes } & Count & 111 & 40 & & \\
\hline & & $\%$ within gender & 68.5 & 24.7 & & \\
\hline \multirow{4}{*}{ Buccal corridor } & \multirow{2}{*}{ Absent } & Count & 106 & 97 & \multirow{4}{*}{1.068} & \multirow{4}{*}{0.301} \\
\hline & & $\%$ within gender & 65.4 & 59.9 & & \\
\hline & \multirow{2}{*}{ Present } & Count & 56 & 65 & & \\
\hline & & $\%$ within gender & 34.6 & 40.1 & & \\
\hline
\end{tabular}

** Significant at 0.01 level; ${ }^{*} P<0.05$, statistically significant.

difference between males and females for the parameter parallelism was statistically significant (Table 2).

3.4. Midline Shift. Midline shift was divided into two categories: $0-1 \mathrm{~mm}$ and $\geq 2 \mathrm{~mm}$ midline shifts (Table 1). $56.2 \%$ of males and $62.3 \%$ of females had their dental midline shifted $2 \mathrm{~mm}$ or above. A chi-square test was applied to detect the difference between females and males in the distribution of midline shift, which showed no statistically significant difference (Table 2).

3.5. Association between Smile Parameters. Spearman's rho correlation test for nonparametric data was applied to investigate the association between the four different variables. Results show a statistically significant positive weak relation between the midline shift and the buccal corridor (" $\rho$ " (rho) $=0.139, P<0.05)$. There was no other statistically significant association between smile parameters (Table 3).

\section{Discussion}

Currently, few criteria are used for smile analyses of young individuals $[6,7,10,12,24,25]$. The purpose of this study was to evaluate and analyse dentogingival macro-aesthetic elements of the smile of young adults within Al Qassim Province. Although limited research has described the smile components of young adults in Saudi Arabia, research has yet to be conducted within $\mathrm{Al}$ Qassim region $[6,7]$.

Tjan et al. discussed three types of smile lines: a high smile line, which reveals the full cervical-incisal length of the anterior maxillary teeth with a contiguous band of the gingiva; an average smile line that reveals $75 \%-100 \%$ of the upper anterior teeth; and a low smile line that reveals less than $75 \%$ of the upper anterior teeth [1]. In the current study, the average smile line was observed to be the most prevalent among participants (seen in $40.4 \%$ of the sample). Other studies have also reported similar findings $[1,12]$. For instance, the smile line was studied by Alqarni et al. within Asser region. These authors found that the average smile line was more commonly seen, compared to a high or low smile line [7]. Nold et al. also investigated the smile line position among Turkish populations. They concluded that a high smile line was a common feature seen in females, while a medium smile line was mostly seen in males [26]. In the current study, we found that a medium smile line was more prevalent in females and a high smile line was more prevalent in males (51.2\% and $41.4 \%$, respectively).

The influence of buccal corridors on smile aesthetics was studied by Albwardi et al. They concluded that excessive teeth display with $2 \%$ buccal corridors was considered to be interpreted as the least attractive smile. However, a medium broad smile with $10 \%$ buccal corridor was considered as the most attractive [27]. Liang et al. also studied buccal corridors in 188 Chinese females and males and found that the buccal corridor was present in $69 \%$ and $51.1 \%$, respectively [25]. Interestingly, in the present study, the buccal corridor was absent in $65.4 \%$ of females and $59.9 \%$ of males.

The incisal curve is known to be ideal when the convex curve follows the concavity of the lower lip when smiling [28]. The phrase consonant used to describe this parallel relationship, a nonconsonant or flat, smile arc can be seen 
TABLE 3: Correlation matrix for the association between smile parameters.

\begin{tabular}{lccccc}
\hline & & Midline shift & Lip line & Parallelism & Buccal corridor \\
\hline & Midline shift & 1.000 & $0.021-$ & $0.062-$ & $0.139^{*}$ \\
Spearman's rho & Lip line & - & 1.000 & 0.059 & 0.027 \\
& Parallelism & - & - & 1.000 & 0.033 \\
& Buccal corridor & - & - & - & 1.000 \\
\hline
\end{tabular}

${ }^{*}$ Correlation is significant at the 0.05 level (2-tailed).

when the maxillary incisal curve is flatter than the curve of the lower lip when smiling [29]. Soares et al. concluded that a straight and convex incisal curve was more common than a reverse incisal curve [28]. Moreover, Al-Johany et al. studied 50 participants and found that $78 \%$ had an incisal curve that was parallel with the lower lip [30]. With that said, we found that only $24.7 \%$ of male participants had a parallel smile, with a higher percentage in females $(68.5 \%)$. This difference in the parallel smile between male and female participants was statistically significant. These results were consistent with Nold et al. who concluded that there was a statistical difference between genders in the distribution of the incisal curve, with the parallel smile more commonly seen in females rather than males [26].

The midline is a crucial vertical reference line [31]. Miller et al. studied midline discrepancy and found that, in $70 \%$ of cases, the dental midline coincides with the facial midline [19]. Moreover, these authors stated that minimal deviations in the midline do not affect overall aesthetics. Al-Balkhi and Zahrani found that the prevalence of the midline shift was $30.7 \%$ [31]. However, we found that $51.6 \%$ of males had more than or equal to a $2 \mathrm{~mm}$ midline shift, and $52 \%$ of female participants had more than or equal to a $2 \mathrm{~mm}$ midline shift. We assessed the dental midline by using central incisors as a reference point, while the facial midline was assessed by using the philtrum as a reference point. This approach is considered a valid method for evaluating the midline shift [19].

A limitation of the present study is the limited sample size taken from a single province in Saudi Arabia. The inclusion of a larger representative sample from multiple regions in Saudi Arabia would help investigate whether there is a statistical difference between different regions in terms of the distribution in smile parameters. Further facial reference points were needed for facial midline determination to precisely measure the amount of midline shift. However, cultural principles and religious considerations precluded taking any wider photographs, especially regarding female subjects. Incorporation of additional smile parameters in future research, such as the geometry, proportion, and shade of the anterior teeth, can be beneficial.

\section{Conclusion}

The present study aimed to analyse the smiling components among young adults within $\mathrm{Al}$ Q assim region by evaluating typical smile parameters. We found that the parallelism of the smile arc and lower lip line was statistically higher in females than males in $\mathrm{Al}$ Qassim region. Additionally, there was a statistically significant difference between genders in the position of the lip line. These results underline the importance of smile parameters when restoring a patient's intramural harmony. Moreover, in order to obtain adequate results in oral rehabilitation, it is necessary to take an individual approach to assess each patient while taking into account their own expectations and preferences.

\section{Data Availability}

Due to the ethical and legal responsibility to respect participants' rights to privacy and to protect their identity, the clinical dataset is not publicly available.

\section{Conflicts of Interest}

The authors declare that they have no conflicts of interest.

\section{Acknowledgments}

The authors would like to express their gratitude to the College of Dentistry, Al Qassim University, for supporting them during research conduction. The authors would also like to thank Dr. Souad Alfouzan for her assistance in statistical analysis.

\section{References}

[1] A. H. L. Tjan, G. D. Miller, and J. G. P. The, "Some esthetic factors in a smile," The Journal of Prosthetic Dentistry, vol. 51, no. 1, pp. 24-28, 1984.

[2] D. Omar and C. Duarte, "The application of parameters for comprehensive smile esthetics by digital smile design programs: a review of literature," The Saudi Dental Journal, vol. 30, no. 1, pp. 7-12, 2018.

[3] C. Rufenacht, Principles of Esthetic Integration/Claude R. Rufenacht. SERBIULA (Sistema Libr. 2.0, Quintessence Publishing, Chicago, USA, 2020.

[4] P. F. Johnson, "Racial norms: esthetic and prosthodontic implications," The Journal of Prosthetic Dentistry, vol. 67, no. 4, pp. 502-508, 1992.

[5] N. Sharma, S. F. Rosenstiel, H. W. Fields, and F. M. Beck, "Smile characterization by U.S. white, U.S. Asian Indian, and Indian populations," The Journal of Prosthetic Dentistry, vol. 107, no. 5, pp. 327-335, 2012.

[6] N. A. AlQahtani, S. B. Haralur, M. AlMaqbol, A. J. AlMufarrij, A. A. Al Dera, and M. Al-Qarni, "Distribution of smile line, gingival angle and tooth shape among the Saudi Arabian subpopulation and their association with gingival biotype," Journal of International Society of Preventive \& Community Dentistry, vol. 6, no. Suppl 1, pp. S53-S58, 2016.

[7] M. A. Alqarni, R. A. Almnea, W. S. Asiri, K. D. Alhendi, and N. A. AlQahtani, "Evaluation of smile line in relation to age 
among Saudi population in Asser region," World Journal of Dentistry, vol. 5, no. 3, pp. 157-161, 2014.

[8] N. Bhat, S. S. Mantri, G. V Iliev et al., "First impression of teeth design on others: a facial and personality analysis in the Central Indian population. Niger," International Journal of Clinical Practice, vol. 22, pp. 1503-1508, 2019.

[9] T. B. Shetty, F. Beyuo, and N. H. F. Wilson, "Upper anterior tooth dimensions in a young-adult Indian population in the UK: implications for aesthetic dentistry," British Dental Journal, vol. 223, no. 10, pp. 781-786, 2017.

[10] N. Grover, D. N. Kapoor, S. Verma, and P. Bharadwaj, "Smile analysis in different facial patterns and its correlation with underlying hard tissues," Progress in Orthodontics, vol. 16, p. 28, 2015.

[11] S. A. Al-Saleh, D. A. Al-Shammery, N. A. Al-Shehri, and E. M. Al-Madi, "Awareness of dental esthetic standards among dental students and professionals," Clinical, Cosmetic and Investigational Dentistry, vol. 11, pp. 373-382, 2019.

[12] M. Khan, S. M. R. Kazmi, F. R. Khan, and I. Samejo, "Analysis of different characteristics of smile," BDJ Open, vol. 6, p. 6, 2020.

[13] V. Mollabashi, M. Abolvardi, M. Akhlaghian, and M. I. Ghaffari, "Smile attractiveness perception regarding buccal corridor size among different facial types," Dental and Medical Problems.vol. 55, pp. 305-312, 2018.

[14] T. Moore, K. A. Southard, J. S. Casko, F. Qian, and T. E. Southard, "Buccal corridors and smile esthetics," American Journal of Orthodontics and Dentofacial Orthopedics, vol. 127, no. 2, pp. 208-261, 2005.

[15] S. Nimbalkar, Y. Y. Oh, R. Y. Mok, J. Y. Tioh, K. J. Yew, and P. G. Patil, "Smile attractiveness related to buccal corridor space in 3 different facial types: a perception of 3 ethnic groups of Malaysians," The Journal of Prosthetic Dentistry, vol. 120, no. 2, pp. 252-256, 2018.

[16] S. K. Pisulkar, R. Agrawal, V. Belkhode, S. Nimonkar, A. Borle, and S. R. Godbole, "Perception of buccal corridor space on smile aesthetics among specialty dentist and layperson," Journal of International Society of Preventive and Community Dentistry, vol. 9, pp. 499-504, 2019.

[17] A. M. Alarabi, G. F. Revie, and D. R. Bearn, "Quantification of maxillary dental midline deviation in $2 \mathrm{D}$ photographs: methodology trial," International Orthodontics, vol. 17, no. 2, pp. 312-323, 2019.

[18] A. S. Bidra, F. Uribe, T. D. Taylor, J. R. Agar, P. Rungruanganunt, and W. P. Neace, "The relationship of facial anatomic landmarks with midlines of the face and mouth," The Journal of Prosthetic Dentistry, vol. 102, no. 2, pp. 94-103, 2009.

[19] E. L. Miller, W. R. Bodden, and H. C. Jamison, "A study of the relationship of the dental midline to the facial median line," The Journal of Prosthetic Dentistry, vol. 41, no. 6, pp. 657-660, 1979.

[20] A. Al Taki, A. M. Hamdan, Z. Mustafa, M. Hassan, and S. Abu-Alhuda, "Smile esthetics: impact of variations in the vertical and horizontal dimensions of the maxillary lateral incisors," European Journal of Dentistry, vol. 11, no. 4, pp. 514-520, 2017.

[21] Z. Jafri, N. Ahmad, M. Sawai, N. Sultan, and A. Bhardwaj, "Digital Smile Design-An innovative tool in aesthetic dentistry," Journal of Oral Biology and Craniofacial Research, vol. 10, no. 2, pp. 194-198, 2020.

[22] P. A. Brunton, J. Ratnayake, C. Loch, A. Veerasamy, P. Cathro, and R. Lee, "Indirect restorations and fixed prosthodontics: materials and techniques used by general dentists of New Zealand," International Journal of Dentistry, vol. 2019, Article ID 5210162, 6 pages, 2019.

[23] M. F. Sfondrini, M. Debiaggi, F. Zara et al., "Influence of lingual bracket position on microbial and periodontal parameters in vivo," Journal of Applied Oral Science, vol. 20, no. 3, pp. 357-361, 2012.

[24] G. Sethna, H. Parmar, R. Gaikwad, and S. Nabazza, "Objective smile analysis and its relationship with the lip length in an Indian population-An institution based study," Journal of Dental and Medical Sciences, vol. 18, pp. 67-75, 2019.

[25] L.-Z. Liang, W.-J. Hu, Y.-L. Zhang, and K.-H. Chung, "Analysis of dynamic smile and upper lip curvature in young Chinese," International Journal of Oral Science, vol. 5, no. 1, pp. 49-53, 2013.

[26] S. Nold, S. Horvath, S. Stampf, and M. Blatz, "Analysis of select facial and dental esthetic parameters," The International Journal of Periodontics and Restorative Dentistry, vol. 34, no. 5, pp. 623-629, 2014.

[27] M. A. Albwardi, B. A. Albwardi, S. A. Alajlan, B. K. Almohareb, and A. A. Alowairdhi, "Perception of buccal corridorseffect on smile esthetics among saudis: a survey," International Journal of Dental and Health Sciences, vol. 04, p. 13, 2017.

[28] C. J. Soares, R. B. Fonseca, L. R. M. Martins, and M. Giannini, "Esthetic rehabilitation of anterior teeth affected by enamel hypoplasia: a case report," Journal of Esthetic and Restorative Dentistry, vol. 14, no. 6, pp. 340-348, 2002.

[29] D. M. Sarver, "The importance of incisor positioning in the esthetic smile: the smile arc," American Journal of Orthodontics and Dentofacial Orthopedics, vol. 120, no. 2, pp. 98$111,2001$.

[30] S. S. Al-Johany, A. S. Alqahtani, F. Y. Alqahtani, and A. H. Alzahrani, "Evaluation of different esthetic smile criteria," International Journal of Prosthodontics, vol. 24, pp. 64-70, 2011.

[31] K. Al-Balkhi and A. Zahrani, The Pattern of Malocclusion in Saudi Arabian Patients Attending for Orthodontic Treatment of the College of Dentistry, King Saud University, Riyadh, Saudi Arabia, 1994. 